\title{
CEREBRAL HEMIATROPHY IN AN ADULT, WITH HEMIPLEGIA AND APHASIA. ${ }^{2}$
}

BY A. H. HARRINGTON, M. D. AND W. L. WORCESTER, M. D.

It is believed by the authors of this paper that the case about to be reported is of sufficient interest to warrant bringing it before this Society, because, as far as we are able to determine, the pathology as related to the clinical history is somewhat obscure.

The feature in this case, which is of most interest, is indicated by the title of the paper, namely, "Cerebral Hemiatrophy in an Adult, with Hemiplegia and Aphasia "; but, to make a complete clinical picture of the case, it seems best to give briefly the entire history of the patient. The analytical statement of this history is as follows:

Ist. An acute attack of mental disease in early adult life, with complete recovery.

2nd. The maintenance of a normal mental condition for a number of years, followed by an attack of mental disease similar in symptoms to the first.

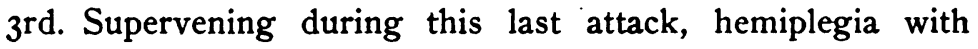
aphasia.

\section{FAMILY HISTORY.}

Patient's father was a steady user of alcoholics, but not given to intoxication. He was reported to have been insane during his life, but the nature of his mental disease could not be ascertained. He is said to have died at the age of 66 , of "Cerebral Apoplexy." One brother of the patient was insane, and confined at one time in the McLean Hospital. One brother had locomotor ataxia.

Patient is said to have had fainting fits when a girl, at which times her face would become flushed.

'Read before the Boston Medico-Psychological Society.

7 
She is said to have been a person of more than ordinary mental capacity, and possessed a cheerful disposition.

CLINICAL HISTORY (BY DR. HARRINGTON).

When twenty-four years of age she was committed to the McLean Hospital, suffering from an attack of mental disease of an acute nature. We are indebted to the McLean Hospital for a full record of the case, the substance of which is as follows:

"This attack began with sleeplessness, and she 'appeared run down,' this being followed by a silly condition, in which she laughed easily, and wrote silly and incoherent love letters to imaginary lovers. She complained of her head feeling badly, and for some time before admission did not seem to recognize her people. While in the hospital, she had outbursts of laughing, crying, and screaming, and was very silly, then became cataleptic - not answering questions, and often bursting into immoderate laughter." She then is said to have had a series of epileptic convulsions. Later on, she is said to have spoken a few words at a time. After a period of four or five months she improved comparatively rapidly, and recovered completely. She was discharged from the McLean Hospital in July, 1873, as " recovered," after a hospital residence of five months.

"She married two years later, and had four children. She was apparently normal up to 1896 , when two children died as a result of accidents-in March and December, respectively, of the year I896. The first death grieved her very much; the second death she took very calmly, but the record says she has not been 'bright' since, and has not slept well. She feared her ' mind would give way.' In December, 1897, one year after the death of her two children, and twenty-four years after her first attack of mental disease, she became depressed; thought she had no clothes; thought that she was the cause of the death of her children ; thought she had not lived right."

In December, 1897 , she was admitted for the second time to the McLean Hospital, going as a voluntary patient.

The record of her history while at the McLean after this second admission is given very fully. I will give the chief features of this record. 
Physical examination on admission was negative, except that the tongue protruded a little to the left, and had a fibrillary tremor. There was considerable tremor of the facial muscles. She was evidently self-condemnatory and depressed. She would stand and gaze in one direction for a long time. She uttered somewhat confused delusions of a depressive nature, but often smiled. She answered somewhat reluctantly, but did not speak slowly. She knew where she was, and knew the time. She read fluently, and remembered fairly well what she had read. She gave age and birthday correctly. In the evening she told correctly what she had for dinner. She counted quickly, but stumbled over simple mathematical problems.

Five days after admission to the McLean Hospital she had an attack in which her eyes rolled up, her face turned dark, and then very pale, and then she appeared confused, but not incoherent. Her conversation was of a very depressive nature. After eight or nine days she spoke little, scarcely noticed anybody or anything; pulled at the doorknobs, pushed the patients about. Her attention could not be attracted. She resisted all efforts to care for her.

A few days later she was noted as staggering around, afterward the record says she almost fell, and looked very exhausted. She still continued to resist and became untidy. She grew emaciated after a time, and did not speak. She repeatedly gave evidence that she understood very well, and one day did everything the nurse asked her to do. From March, 1898, until June, 1898 , she did not speak or answer questions, but lay in bed, and at times showed well marked " muscular negativism."

On August 15, eight months after her second admission to the McLean Hospital, she had a series of convulsions, commencing in the muscles of the left side of the face, then on the right side, then alternating between the right and left sides; at times, the whole body twitching. The convulsions continued for two hours-the last hour of which they were wholly confined to the right side.

A few days later, it was noticed that there was no difference in the nutrition of the two arms; the right was completely paralyzed, so that it fell flaccidly, whereas with the left arm the joints showed well-marked muscular negativism, and at times distinct 
catalepsy. There was a slight difference in the two facial halves, the right nasolabial fold appeared somewhat flatter. The patient cannot be made to perform any voluntary motions. When lying quietly, the upper lip on the left side hangs higher than on the right, so that the nasolabial fold is deeper. The forehead is equal on both sides. On the right side, between the eyelids, there is a larger gap than on the left, so that while the left eyelid covers a part of the iris, there is frequently seen a portion of the sclera above the iris, on the right, and this becomes distinctly marked when the patient looks down. There is inequality in the movement of the two eyes. The kneejerk on the left is difficult to obtain because the patient resists; on the right side it is well marked. No ankle or patella clonus. Plantar reflexes present on the left side, but not on the right. There is wellmarked dermographia on both sides. Electrical examination negative. Patient did not react to prick of pin on either side.

In October, although the muscles of the right arm had atrophied, no change was found on electrical examination. The patient seemed to feel the strong currents, as she cried out more markedly. After the first interosseus of the right hand had been tested, the index finger of that hand made a rapid flexion and extension movement of short excursion, chiefly in the metacarpophalangeal joint. This was repeated several times. Upon electrical examination, the arm was moved distinctly in the elbow and wrist joint.

In November, it was noticed that she continued to move her arm at the elbow and wrist as described above, but with no perceptible change. Her hand was held with fingers flexed upon the hand and partial flexion at the elbow, and she could move her arm voluntarily to some extent.

She continues to remain in bed, never speaks coherently, frequently shouts at the top of her voice, and is very untidy.

On May 25, 1899, she was transferred to the Danvers Insane Hospital.

Examination on admission to the Danvers Hospital was not at first satisfactorily made on account of the restless condition of the patient. It is noted that the arteries were not atheromatous. She paid no attention to examiner's questions, and kept stuttering and repeating such words as $\mathrm{Ma}$ ! Ma! Ma! Sa! Sa! Sa! and other jargon. 
May 30, two and one-half months after admission, the following notes were made:

There is no apparent facial paralysis. Tongue deviates somewhat to the right. Right arm and hand seem partially contractured. In walking, she keeps her right knee partially flexed, and it has a tendency to give way under her. She appears to understand some directions; gives her hand when asked to, and opens her mouth when asked to show her tongue, but cannot be induced to put her hand on her head.

Occasionally, she speaks a few intelligible words, and said in reply to a direction, "I can't," twice. Said, " Oh! I don't know," and "Well, I guess." Will sometimes repeat a word that is said to her, but most of her vocal sounds are meaningless syllables repeated over and over. Nurse reports that she seems to have difficulty in swallowing.

July 3r. Screams and makes inarticulate sounds a great deal of the time. She appears to know the names of some of the nurses, and will repeat them over and over. Will at times repeat other words. Yesterday, read the name "Sarah" on a nurses apron, and kept repeating it for some time. Soils and wets her clothing.

During the next three months of her life she had three or four attacks of general convulsions, finally a series of convulsions were followed by her death.

The clinical diagnosis of the case now remains to be considered.

It is evident that at the age of twenty-four, the patient had an acute psychosis. This attack appears from the clinical account to have been of the maniacal-depressive type with manifestations of the katatonic symptom complex, as evidenced in the mutism and the cataleptic state.

After recovery and twenty-four years of mental health, if the history given by her family is correct, as I believe from personal interview that it was, there appears to have been a recurrence of mental symptoms similar to the first attack. There was mental depression and confusion. We observe also in this second attack katatonic manifestations, as it is noted that from March to June of 1898 , the patient did not speak, but lay in bed and showed "muscular negativism." Then, about two months later, 
occurred a series of convulsions followed by an unmistakable hemiplegic condition of the right side and pronounced aphasia.

It will be remembered that during the latter part of the first attack, there were convulsions, but during the twenty-four years of mental health which followed, nothing of an epileptic nature could be ascertained.

We are not warranted, it seems to me, in making any positive assertion as to the bearing of the first and second attacks upon each other, beyond saying that the early portion of the second attack bore a close resemblance to the first, and we are reminded of the possibility of the recurrence of a psychosis. The latter manifestations of the second attack, however, place the case for the final months of the patient's life in the group of organic diseases. The possibility of general paralysis has to be considered, but the hemiplegia and aphasia are against its being a clear case of that disease.

The diagnosis for the last of the patient's life, which was determined and recorded, was "post-paralytic dementia with hemiplegia and aphasia."

$$
\text { AUTOPSY (BY DR. WORCESTER). }
$$

At the autopsy, the findings as regards the nervous system were as follows:

Nothing of interest noted in regard to cranium and dura.

Pia-arachnoid cloudy and œdematous; separated readily from brain.

Cerebral vessels not atheromatous. No obstruction discovered on careful inspection.

Right hemisphere presented no noticeable abnormality.

Left hemisphere considerably smaller than the right, with narrow convolutions and gaping sulci. This was specially noticeable in the frontal and temporal lobes. The island of Reil was partly exposed by their retraction. The cortex of this hemisphere appeared unusually friable, and its superficial layer, although nowhere adherent to the membranes, was abraded in spots in handling it. A little behind the upper end of the posterior central gyrus was a small discolored spot, appearing as if due to superficial necrosis. 
THE AMERICAN JOURNAL OF INSANITY, Vol. LVIII, No. $1 . \quad$ PLATE I.

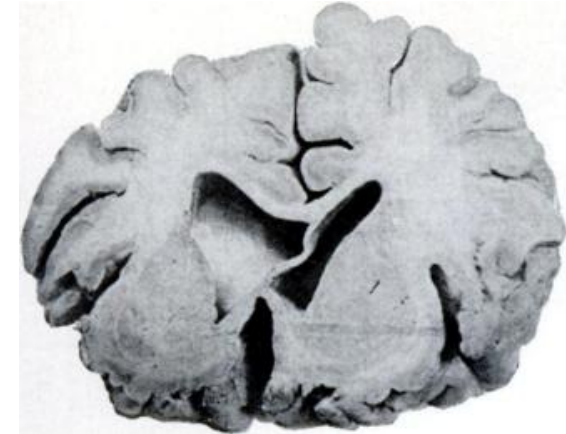

FIG. 1.

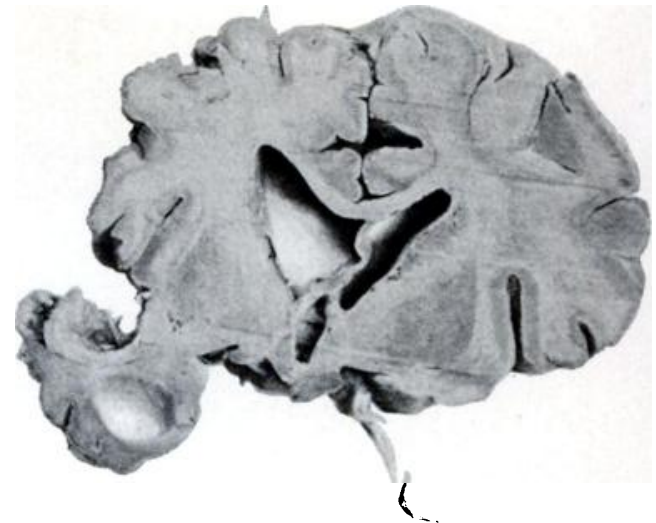

FIG. 2.

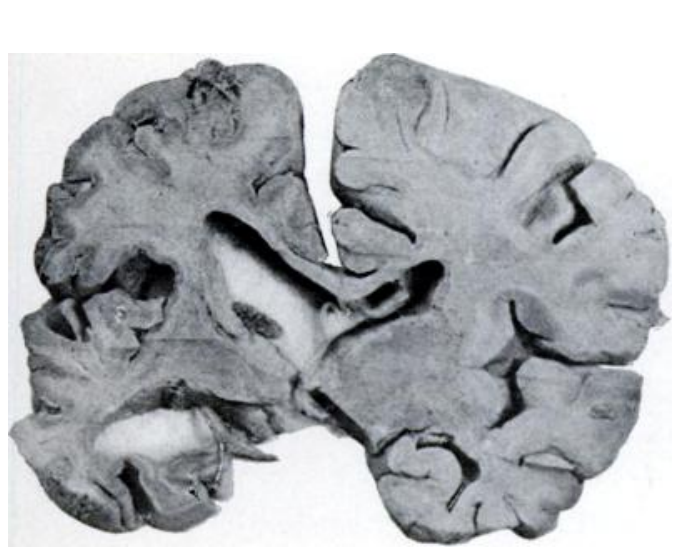

FIG. 3.

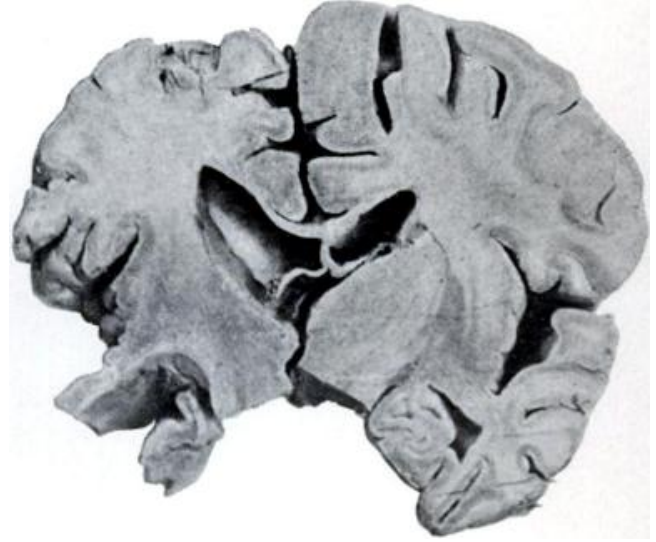

FIG. 4.

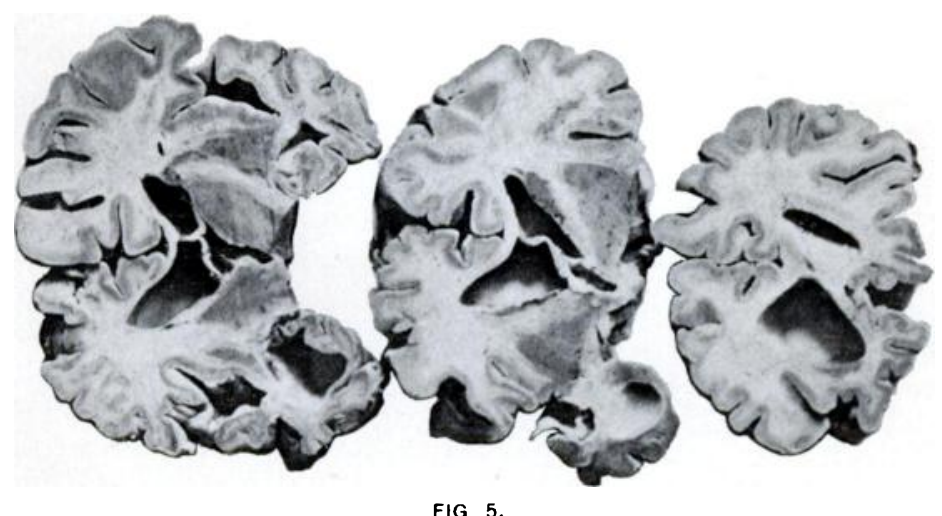

FIG 5. 
THE AMERICAN JOURNAL OF INSANITY, Vol. LVIII, No. 1.

PLATE II.

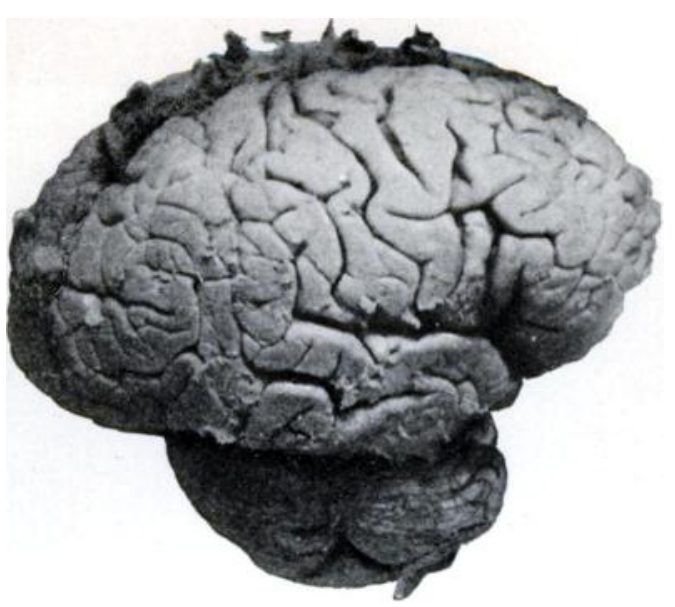

FIG. 6 .

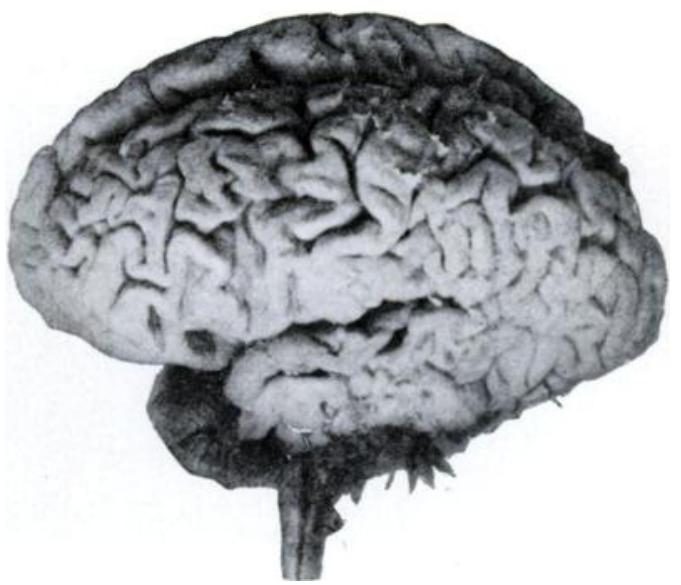

FIG. 7.

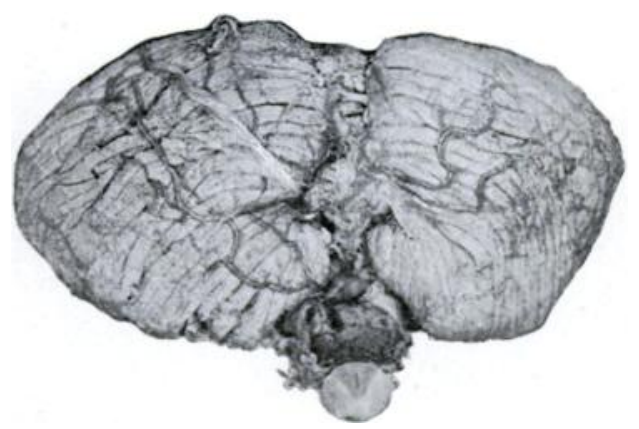

FIG. 8. 
The brain weighed, with the membranes, 973 grammes. It was hardened in formalin before dissection. The following additional facts were then ascertained:

The right hemisphere weighed 445 , the left, 342 grammes.

The left lateral ventricle was very greatly dilated in its entire extent.

For about the middle third of the posterior branch of the fissure of Sylvius, the cortex of the inner aspect of the first left temporal convolution and of the lower half of the island of Reil was greatly atrophied, and over a portion of this area its superficial portion separated when the section was made. No focus, either of hæmorrhage or softening, was found in the interior of the brain.

There was no marked asymmetry of the corpora striata, but the left optic thalamus was much smaller than the right.

The right hemisphere of the cerebellum was very much smaller than the left. The apparent disproportion was even greater than in the cerebrum. The accompanying photographs afford good illustrations of these various points.

Nothing noticeably abnormal was discovered on inspection of the spinal cord.

On microscopical examination of the region already mentioned, in the left fissure of Sylvius, the cortex appears very greatly atrophied, and it is evident that the nerve-cells have, to a large extent, disappeared. They are still to be seen, however, in considerable numbers, greatly atrophied; in many cases only the nuclei are distinguishable. The cortex is greatly diminished in thickness. There is no apparent hypertrophy of neuroglia in the cortex, and its blood-vessels are not conspicuously altered. In the underlying white substance, spider-cells are to be seen in considerable numbers, and many of the vessels are tortuous. There is nothing suggestive of a deficient blood supply.

In sections from corresponding portions of the cortex of the two hemispheres elsewhere, the differences in the number and appearance of the nerve-cells are not striking. Owing to thinning of the cortex of the left hemisphere, the cells appear more crowded and in some portions more slender than in the corresponding sections from the right hemisphere. Some peculiar appearances are seen in the nuclei of nerve-cells, of the signifi- 
cance of which I am not satisfied, but they are quite as noticeable in the right as the left hemisphere, and can hardly, I think, be responsible for the atrophy of the latter.

In both cornua Ammonis, the nucleus fasciæ dentatæ are almost destitute of nerve-cells. The stratum pyramidale is not atrophied. Sections from the second frontal gyrus of each side stained with Wolters' modification of the Weigert stain show no marked difference in the numbers of medullated fibres in the cortex.

The shrinkage appears to be more at the expense of the white than the gray matter. There is no conspicuous proliferation of neuroglia in the cortex of the portions examined.

Specimens from both lateral lobes of the cerebellum show a great diminution in number of the Purkinjean cells. On the right side, they are entirely wanting over large areas; in many cases a pretty large section can be examined without finding one. The few that remain present, for the most part, a normal appearance. The cells of the granular layer are very greatly reduced in number, and in certain areas are almost entirely wanting. The molecular layer is reduced to about half its normal thickness, and appears to be made up mainly of parallel neuroglia fibres, running perpendicularly to its surface. In the medullary portion, the nerve-fibres are much reduced in number, and there is some hypertrophy of neuroglia.

In sections from different parts of the left lateral lobe, the number of Purkinjean cells varies considerably, although they appeared to be nowhere present in anywhere nearly normal abundance, and are sometimes entirely absent over large areas. Most of those which remain are normal in appearance, although some appear to be in process of atrophy. Apart from the absence of these cells, the tissue is of healthy appearance.

In the medulla and pons, sections stained by Van Gieson's and Wolters' methods show nothing abnormal except a pretty considerable degeneration of the left pyramidal tract. Sections stained by the Marchi method show the presence of a small number of degenerated fibres confined to the same tract. Sections from the spinal cord at various levels seem normal except for a marked degeneration of the crossed pyramidal tract.

To recapitulate: We have, in this case, clinically, right hemi- 
plegia with contractures, and pretty complete aphasia, occurring in a woman previously insane, subsequently to a series of severe convulsive seizures. Anatomically, there was atrophy of the entire left hemisphere of the cerebrum and of the pyramidal tract connected with it, as well as of the opposite hemisphere of the cerebellum. There was no evidence of hæmorrhage or of arterial obstruction, nor anything which could be considered a focal lesion, although the changes appeared to be more marked in the fissure of Sylvius, the optic thalamus and the cerebellum than elsewhere.

In view of the previous symptoms of the patient, it is perhaps pertinent to enquire to what extent her condition was to be attributed to the anatomical lesions. The changes in the central nervous system bore a very strong resemblance to those often found in cases of infantile hemiplegia, and it might be questioned whether they were not actually of long standing.

For my own part, I feel no doubt that the hemiplegia was due to the lesions. To suppose the contrary would require us to believe, first, that these changes had existed during a pretty long life without producing symptoms, and, secondly, that hemiplegia had occurred suddenly without any demonstrable lesion, each of which hypotheses, taken by itself, seems sufficiently improbable, while the combination of the two, to my mind, is scarcely credible. But, furthermore, the recent origin of the anatomical changes is, as it seems to me, put beyond reasonable doubt by the fact that the Marchi stain reveals the presence of degenerated myeline in the pyramidal tract.

It must, I think, be considered doubtful to what extent the patient's dementia and disturbances of speech were attributable to the anatomical changes. She had previously been in a stupid condition, and had gone for pretty long periods without uttering anything but inarticulate sounds. On the other hand, such extensive lesions could hardly be compatible with mental soundness, and the degree of atrophy in and about the fissure of Sylvius would be sufficient to account for almost any degree of aphasia. I can only say that the patient always impressed me as suffering from a very grave organic speech-defect, but that it seems to me probable that her dementia was not entirely due to atrophy of the brain. 
A question of still greater interest and obscurity is that of the nature of the original pathological process. The usual causes of permanant hemiplegia, in adults at least, are hæmorrhage and arterial obstruction, both of which can, I think, be excluded in this case. Strümpell has advanced the hypothesis of a polioencephalitis to account for similar conditions in cases of infantile hemiplegia. It may not be possible to exclude such a process in this case, but I was unable to find any affirmative evidence of it. The appearances are, to my mind, no more suggestive of an inflammatory process than those found in the cerebral atrophy of senile dementia.

In a recent article by Mott and Tredgold, ${ }^{2}$ on Hemiatrophy of the Brain and its Results on the Cerebellum, Medulla and Spinal Cord, the authors take the ground that crossed atrophy of the cerebellum is always due to a primary lesion in the basal ganglia. In my case, as already mentioned, there does not seem to be any marked shrinkage of the nucleus lenticularis. The optic thalamus is markedly diminished in size, but I find no evidence of a focal lesion there, and believe the diminution to be secondary to the changes in the cerebrum, rather than the reverse. The changes in the atrophied lobe of the cerebellum are precisely similar to those described by these authors in one of their cases, but the layer of cells which they describe as lying immediately under the Purkinjean cells in the cerebellar cortex, and as being preserved, while the other cellular elements of the cortex are, to a great extent, destroyed, seems to me to be, unquestionably, neurogliar, not nervous in its nature. The very great deficiency of Purkinjean cells in the opposite lobe of the cerebellum, without any apparent atrophy of either the molecular or the granular layer, is remarkable, and, to me, inexplicable.

The great similarity of this case, both in clinical history and anatomical lesions, to many cases of infantile cerebral paralysis is obvious. It seems to me scarcely doubtful that it is of essentially the same nature, but I have been unable to find, in the literature accessible to me, an account of a similar case occurring in an adult. I have never been able to satisfy myself that the post-mortem appearances found in some of the cases which I

${ }^{1}$ Brain, vol. 23, 1900, p. 239. 
have had the opportunity of examining were satisfactorily accounted for by any of the explanations that I have seen. I cannot claim that my case throws any positive light upon this subject, but it seems to show that neither cerebral hæmorrhage nor arterial obstruction accounts for all the cases. The first step in the solution of a problem is the appreciation that there is a problem to be solved. So long as we rest satisfied with erroneous explanations, we shall look no further. Reports of careful post-mortem examinations of recent cases of this kind are much to be desired. 